\title{
PSMD12 promotes glioma progression by upregulating the expression of Nrf2
}

\author{
Zhongyong Wang ${ }^{1 \#}$, Zhiyu $\mathrm{Li}^{2 \#}$, Hui Xu ${ }^{1 \#}$, Yun Liao ${ }^{1}$, Chao Sun ${ }^{1}$, Yanming Chen ${ }^{1}$, Minfeng Sheng ${ }^{1}$, \\ Qing Lan ${ }^{1}$, Zhong Wang ${ }^{2}$
}

${ }^{1}$ Department of Neurosurgery, The Second Affiliated Hospital of Soochow University, Suzhou, China; ${ }^{2}$ Department of Breast and Thyroid Surgery, Renmin Hospital of Wuhan University, Wuhan, China

Contributions: (I) Conception and design: Z Wang, Z Li, Z Wang; (II) Administrative support: Q Lan, Z Wang; (III) Provision of study materials or patients: Z Wang, Z Li, H Xu; (IV) Collection and assembly of data: Z Wang, Z Li, H Xu, Y Liao, C Sun, Y Chen, M Sheng; (V) Data analysis and interpretation: Z Wang, Z Li, Z Wang; (VI) Manuscript writing: All authors; (VII) Final approval of manuscript: All authors.

\#These authors contributed equally to this work.

Correspondence to: Zhong Wang. Department of Breast and Thyroid Surgery, Renmin Hospital of Wuhan University, 99 Zhang Zhidong Road, Wuhan 430060, China. Email: zhongwangchn@whu.edu.cn; Qing Lan. Department of Neurosurgery, The Second Affiliated Hospital of Soochow University, 1055 Sanxiang Road, Suzhou 215004, China. Email: szlanqing123@163.com.

Background: Glioma is the most common and aggressive primary brain tumor in adults. Proteasome $26 \mathrm{~S}$ subunit, non-ATPase 12 (PSMD12), an important subunit in the 26S proteasome, is known to be involved in the growth and apoptosis of breast cancer cells. However, its exact function and underlying molecular mechanisms in glioma remain unknown.

Methods: PSMD12 expression was detected in glioma tissue specimens by immunohistochemistry (IHC) and TCGA database. Overexpression and down-regulation of PSMD12 and Nrf2 were induced in glioma cell lines, and CCK-8 and Transwell assays were used to detect cell proliferation and invasion evaluation, respectively. Xenograft model was used to observe the effect of knockdown of PSMD12 on tumor growth. Immunohistochemical assays and TCGA database were conducted to reveal the relationships between PSMD12 expression and Nrf2. Finally, Western blot and related biological function experiments were used to explore the mechanism of PSMD12 regulating the glioma progression and Nrf2.

Results: We revealed that PSMD12 is upregulated in glioma, especially in high-grade glioma, by analyzing bioinformatics data and clinical specimens. PSMD12 upregulation was associated with poor prognosis in glioma patients. Knockdown of PSMD12 inhibited the growth of glioma cells in vitro and in vivo and decreased their invasion ability, whereas PSMD12 overexpression had the opposite effect. Mechanistic analysis revealed that PSMD12 increased the expression of nuclear factor E2-related factor 2 (Nrf2), which functions as a tumor promoter in the development of glioma. Similar to PSMD12, Nrf2, which exhibited a strong positive correlation with PSMD12, was abnormally elevated in glioma tissues and contributed to worse overall survival (OS). Nrf2 overexpression reversed the inhibitory effects induced by PSMD12 knockdown. Finally, PSMD12 enhanced the proliferation and invasion of glioma cells via Akt signalingmediated Nrf2 expression.

Conclusions: These results suggest that PSMD12 is considered to be a crucial regulator of the development and progression of glioma and may serve as a potential biomarker or therapeutic target for the treatment of glioma.

Keywords: Proteasome 26S subunit; non-ATPase 12; glioma; cell survival; nuclear factor E2-related factor 2 (Nrf2); Akt pathway

Submitted Mar 01, 2021. Accepted for publication Apr 23, 2021.

doi: 10.21037/atm-21-1481

View this article at: http://dx.doi.org/10.21037/atm-21-1481 


\section{Introduction}

Glioma is the most common intracranial carcinoma, accounting for $81 \%$ of brain tumors (1). The most frequent histological type is glioblastoma (GBM), which accounts for $65 \%$ of all glioma (2). The median overall survival (OS) time of GBM patients is only 12-18 months (3). Glioma feature rapid growth, invasiveness, and resistance to therapy. Currently, maximal surgical resection, external beam radiation, and chemotherapy are the main treatments for GBM. However, side-effects and drug resistance are unavoidable, and new therapeutic targets remain to be identified.

Proteasome 26S subunit, non-ATPase 12 (PSMD12; also known as RPN5) was first discovered in fission yeast (4). It is a non-ATPase subunit of the $19 \mathrm{~S}$ regulator of the $26 \mathrm{~S}$ proteasome complex, which is involved in regulating the cell cycle, repairing DNA damage, and regulating apoptosis by mediating the internal protein balance through the removal of misfolded proteins or damaged intracellular substructures $(5,6)$. Given that neurons are vulnerable to ubiquitin-proteasome system (UPS) dysfunction, deletion of PSMD12 can cause neurodevelopmental disorders (5). Moreover, it has been reported that PSMD12 is required for enzalutamide resistance in castration-resistant prostate cancer (7). In addition, PSMD12 contributes to tumor cell growth in breast cancer (8). Therefore, it seems that PSMD12 can promote cancer progression.

Nuclear factor E2-related factor 2 (Nrf2) is a transcription factor that is composed of 7 NRF2-ECH homology domains. It is capable of activating cytoprotective genes and can regulate the cellular defense against xenobiotics and oxidative stress (9). Nrf2 is also involved in autophagy and the unfolded protein response (9). Nevertheless, persistent activation of Nrf2 in tumor cells contributes to resistance against anticancer therapies and reactive oxygen species and promotes metabolic reprogramming (10). Nrf2 is reported to be hyperactivated in GBM and anaplastic glioma $(11,12)$. The upregulation of Nrf2 is related to poor prognosis in glioma patients $(11,12)$. Its overexpression in glioma cells accelerates their proliferation, invasion, and mesenchymal transition $(11,13)$.

In the present study, we explored the expression levels of PSMD12 and Nrf2 in glioma and their effects on prognosis by bioinformatics analysis and immunohistochemical staining. We further investigated the function of PSMD12 and Nrf2 in glioma and further explored the molecular mechanism by which PSMD12 regulates Nrf2. The aim of the present study was to provide a novel antitumor therapeutic target for patients with glioma.

We present the following article in accordance with the ARRIVE reporting checklist (available at http://dx.doi. org/10.21037/atm-21-1481).

\section{Methods}

\section{Data acquisition}

PSMD12 expression in glioma patients and its association with OS were analyzed using the Gene Expression Profiling Interactive Analysis (http://gepia.cancer-pku. $\mathrm{cn} /$ ), GlioVis (http://gliovis.bioinfo.cnio.es/), and Chinese Glioma Genome Atlas (CGGA; http:// http://cgga.org.cn/) websites.

\section{Ethics statement}

Glioma tissues and normal brain tissues were collected at the Second Affiliated Hospital of Soochow University. All procedures performed in this study involving human participants were in accordance with the Declaration of Helsinki (as revised in 2013). The study was carried out in accordance with the principles of the Ethics Committee of Second Affiliated Hospital of Soochow University. All human participants involved in this study provided informed consent. The animal experiment complied with the principles of the Animal Centre of the Second Affiliated Hospital of Soochow University.

\section{Cell culture and reagents}

The glioma cell lines GBM12 and U251 were purchased from American Type Culture Collection (ATCC; Rockville, MD, USA). They were cultured in Dulbecco's modified Eagle's medium (Gibco, New York, USA) supplemented with $10 \%$ fetal bovine serum and incubated at $37{ }^{\circ} \mathrm{C}$ with $5 \% \mathrm{CO}_{2}$. The Akt inhibitor MK2206 was purchased from Selleck (S1078, Shanghai, China) and diluted in dimethyl sulfoxide.

\section{Immunobistochemistry (IHC)}

IHC was performed according to a previously published study (14). The number of positive cells was scored as follows: $\sim 10 \%=0,10-20 \%=1,21-50 \%=2$, or $50 \% \sim=3$. The intensity was evaluated as follows: $0=$ no staining, $1=$ 
weak staining, $2=$ moderate staining, or $3=$ strong staining. The score for the number of positive cells multiplied by the intensity score represented the final score: $0=$ negative expression, $1-3=$ low expression, or $4-9=$ high expression.

\section{siRNA and plasmid transfection}

siRNAs against PSMD12 (5'-GUGGUGACAAGAAGUUAGATT-3'), Nrf2 (5'-CUUGCAUUAAUUCGGGAUATT-3') and scrambled siRNA (5'-UUCUCCGAACGUGUCACGUTT-3') were obtained from GenePharma Co. (Shanghai, China). Flag-PSMD12, Flag-Nrf2, and Flag-NC (NC acts as a negative control) were purchased from GeneChem Co. (Shanghai, China). siRNAs or plasmids were mixed with Lipofectamine 2000 (Invitrogen, Carlsbad, USA) in reduced serum medium (Opti-MEM; Gibco, USA) according to the manufacturer's instructions. Transient transfections were carried out as described previously (14).

\section{Cell Counting Kit (CCK)-8 assay}

Cells $(2,000$ cells/well) were seeded into 96-well plates and cultured for 24 and $48 \mathrm{~h}$. CCK-8 (Dojindo Molecular Technologies, Gaithersburg, MD, USA) solution was added to the medium and incubated for $2 \mathrm{~h}$ at $37^{\circ} \mathrm{C}$ away from light. The absorbance was measured at $450 \mathrm{~nm}$.

\section{Invasion assay}

Cells subjected to the indicated treatments were seeded in the upper chambers of 24 -well plates at $2 \times 10^{4}$ cells/well in serum-free medium; the chambers were precoated with Matrigel (Corning, New York, USA). Complete medium was added to the lower chamber. After incubation for $24 \mathrm{~h}$, the cells were fixed with formaldehyde, stained with crystal violet, and counted.

\section{Western blotting}

The protocol of Western blotting was based on that of a previously published study (14). The same amounts of proteins were separated by sodium dodecyl sulfatepolyacrylamide gel electrophoresis and transferred to nitrocellulose membranes. The membranes were then blocked in $5 \%$ skim milk for at least $2 \mathrm{~h}$ and incubated with primary antibodies at $4{ }^{\circ} \mathrm{C}$ overnight and secondary antibodies at room temperature for $1 \mathrm{~h}$. Antibodies against PSMD12 (11412-1-AP; Proteintech, China), FLAG
(F1804; Sigma, USA), Nrf2 (16396-1-AP; Proteintech, China), NRBP2 (21549-1-AP; Proteintech, China), p-Akt (4060; Cell Signaling Technology, USA), Akt (2920; Cell Signaling Technology, USA), p-mTOR (5536; Cell Signaling Technology, USA), mTOR (2983; Cell Signaling Technology, USA), and $\beta$-actin (A5441; Sigma, USA) were used.

\section{Animal experiments}

GBM12 cells were transfected with PSMD12 knockdown lentivirus obtained from GeneChem Co. (China) and selected with puromycin. The level of PSMD12 was detected by Western blotting before the injection. Four- to 5-week-old female athymic nude mice (BALB/ c) were randomly divided into two groups. They were subcutaneously injected with $1.5 \times 10^{6}$ stable cells that were resuspended in phosphate-buffered saline and Matrigel (Corning, New York, USA). Tumors were excised or weighed and photographed when they reached a suitable size. The expression of PSMD12, Nrf2, and Ki67 in xenografts was measured by IHC.

\section{Statistical analysis}

Data were presented as the mean \pm standard deviation (SD) from triplicate independent experiments. Data were assessed by SPSS version 24.0 (IBM, Armonk, NY, USA). $\mathrm{P}<0.05$ indicated statistically significant results.

\section{Results}

\section{PSMD12 upregulation in glioma and association with poor prognosis in glioma patients}

To determine the role of PSMD12 in the progression of glioma, we first assessed the expression levels of PSMD12 in normal brain tissues and glioma tissues or glioma tissues of different grades from The Cancer Genome Atlas (TCGA) or CGGA database. We found that PSMD12 was expressed at higher levels in glioma tissues than in normal tissues. Moreover, the expression of PSMD12 was markedly higher in high-grade glioma tissues than in low-grade glioma tissues (Figure 1A,B,C). We then used IHC to detect PSMD12 expression in glioma tissues and normal brain tissues. As shown in Figure 1D, PSMD12 expression was noticeably higher in glioma tissues than in normal tissues, and was also significantly higher in grade IV glioma tissues 


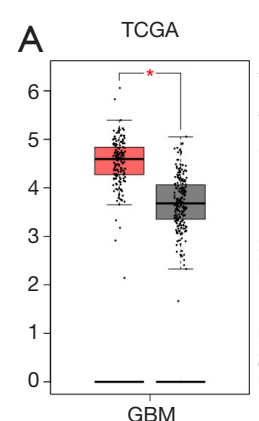

$[\operatorname{num}(T)=163 ; \operatorname{num}(\mathrm{N})=207]$

D
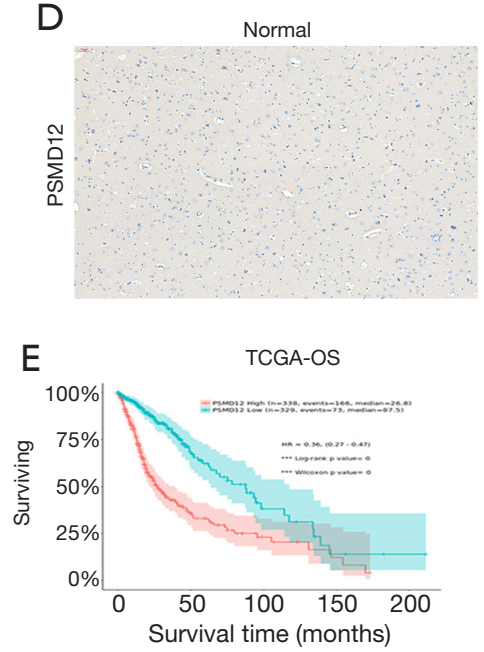

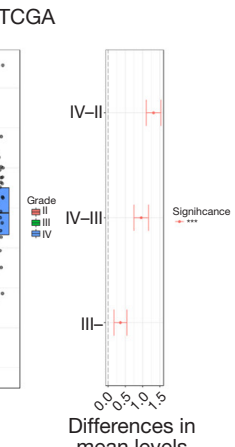

mean levels
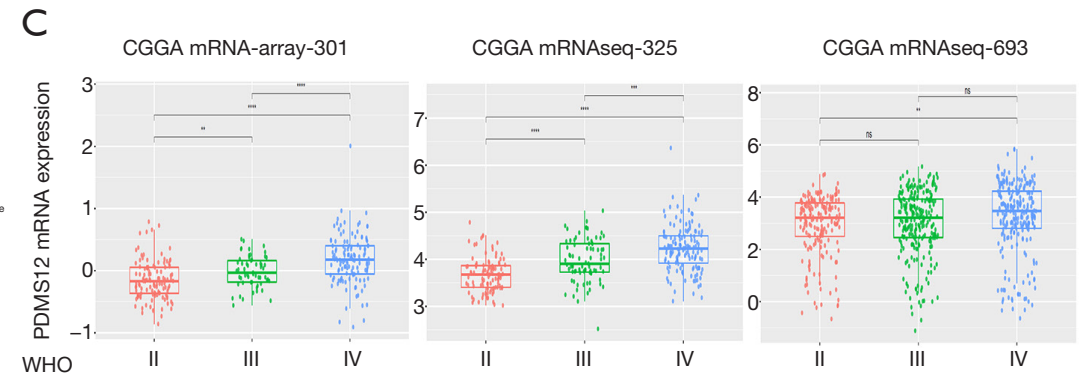

WHO III
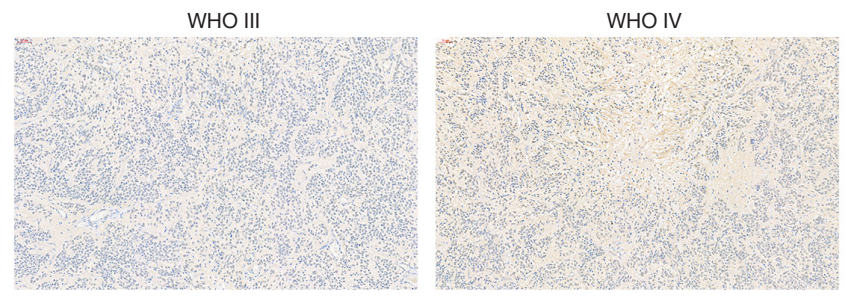

WHO II
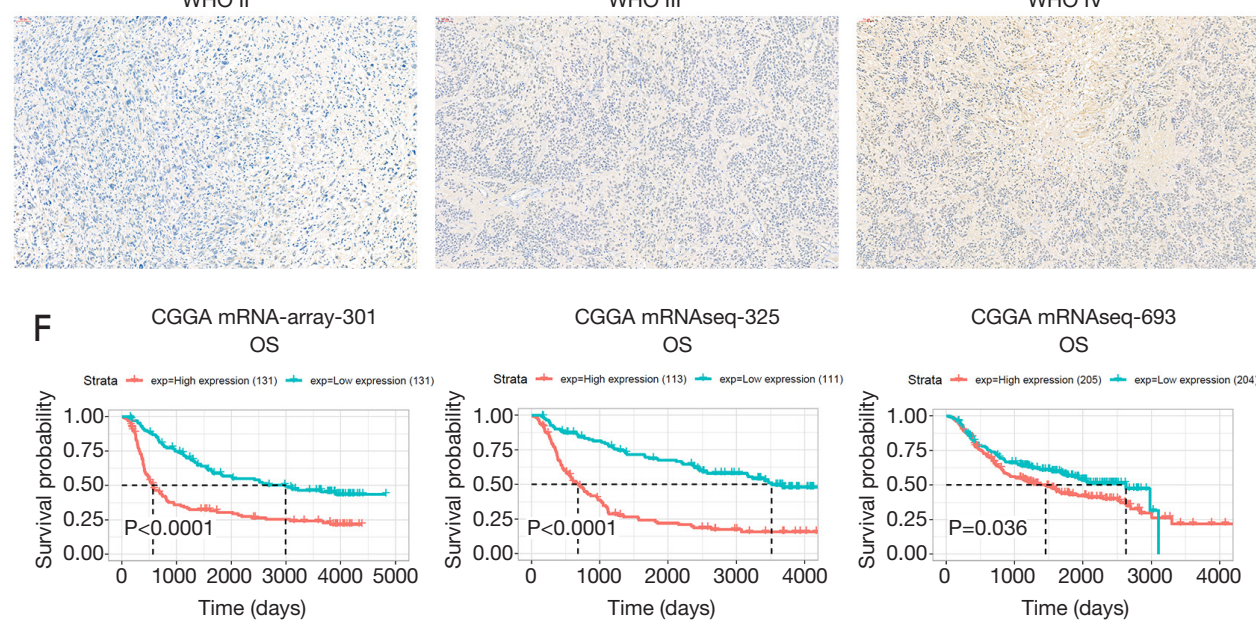

Figure 1 Proteasome 26S subunit, non-ATPase 12 (PSMD12) is upregulated in glioma and associated with poor prognosis in glioma patients. (A,B,C) Relative levels of PSMD12 in glioma tissues and adjacent tissues in The Cancer Genome Atlas (Gene Expression Profiling Interactive Analysis), the GlioVis, and Chinese Glioma Genome Atlas (CCGA) databases. (D) Expression of PSMD12 in glioma tissues and normal tissues by immunohistochemistry (magnification $\times 200$ ). (E,F) Kaplan-Meier analysis showed the overall survival of glioma patients with high/low PSMD12 expression from the GlioVis and CGGA databases. *, $\mathrm{P}<0.05$; **, $\mathrm{P}<0.01$; and ${ }^{* * *}, \mathrm{P}<0.001$ compared with the control group.

than in grade II or III glioma tissues. Furthermore, KaplanMeier analysis of the OS of patients in TCGA and the CGGA databases showed that patients with high levels of PSMD12 had significantly poorer prognoses than patients with low levels of PSMD12 (Figure 1E,F). Taken together, these results suggest that PSMD12 is upregulated in glioma, and that high PSMD12 expression is an unfavorable prognostic factor in terms of OS.

\section{PSMD12 promotes the growth and invasion of glioma cells in vitro}

To further evaluate the role of PSMD12 in glioma cell biological functions, siRNA or the FLAG-PSMD12 plasmid was applied to decrease or increase the expression of PSMD12, respectively, in glioma cells. Western blotting results in GBM12 and U251 cells showed that the expression level of PSMD12 was reduced by at least $80 \%$ in cells transfected with the siRNA, and that PSMD12 was significantly overexpressed in glioma cells transfected with the plasmid (Figure 2A,B). CCK-8 cell growth assays showed that the knockdown of PSMD12 significantly impaired GBM12 and U251 cell proliferation, while the overexpression of PSMD12 markedly promoted the growth of both glioma cell lines (Figure 2C,D). In addition, cell invasion ability decreased after knockdown of PSMD12 in GBM12 and U251 cells, and increased with PSMD12 overexpression in both glioma cell lines (Figure 2E,F). Therefore, these findings suggest that PSMD12 enhances the proliferation and invasion ability of glioma cells in vitro. 
A

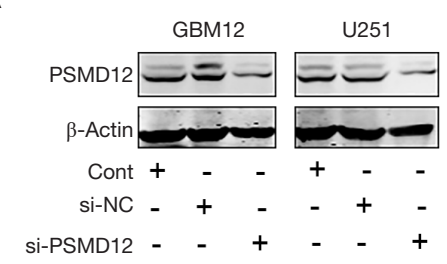

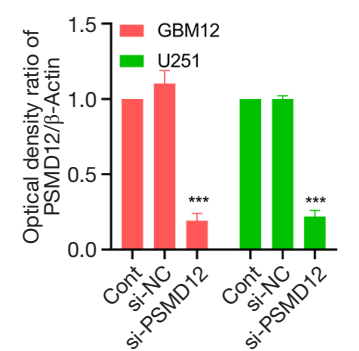

U251
B

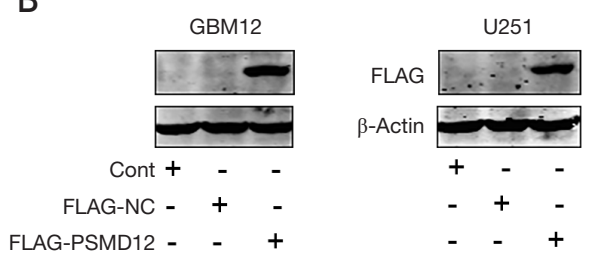

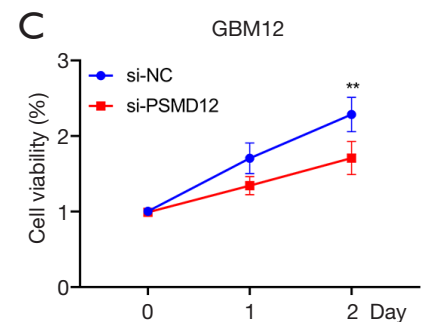

E

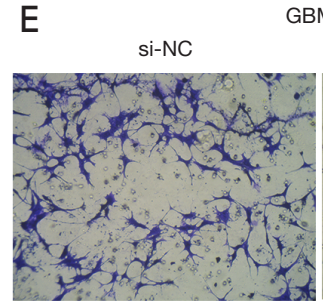

GBM12

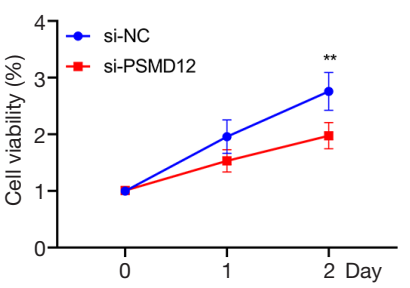

D

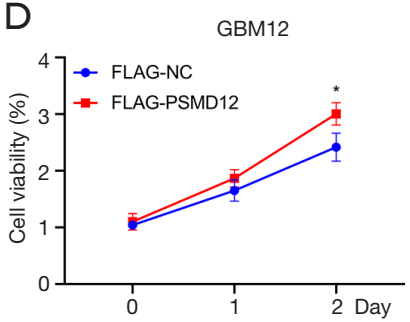

U251

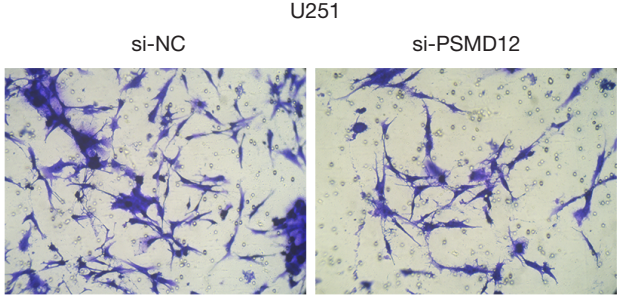

U251

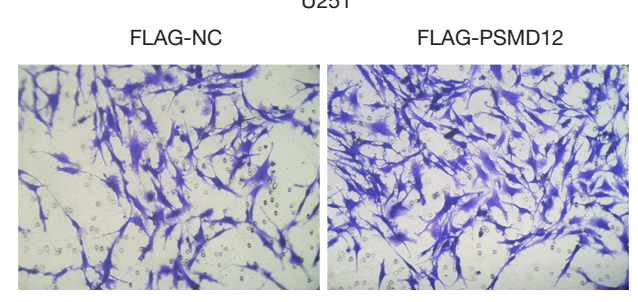

si-PSMD12

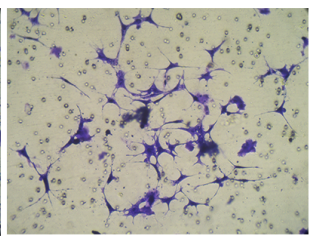

GBM12

FLAG-PSMD12

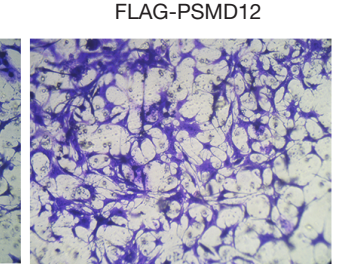

FLAG-NC

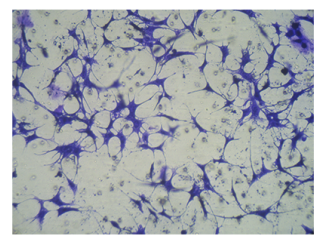

U251
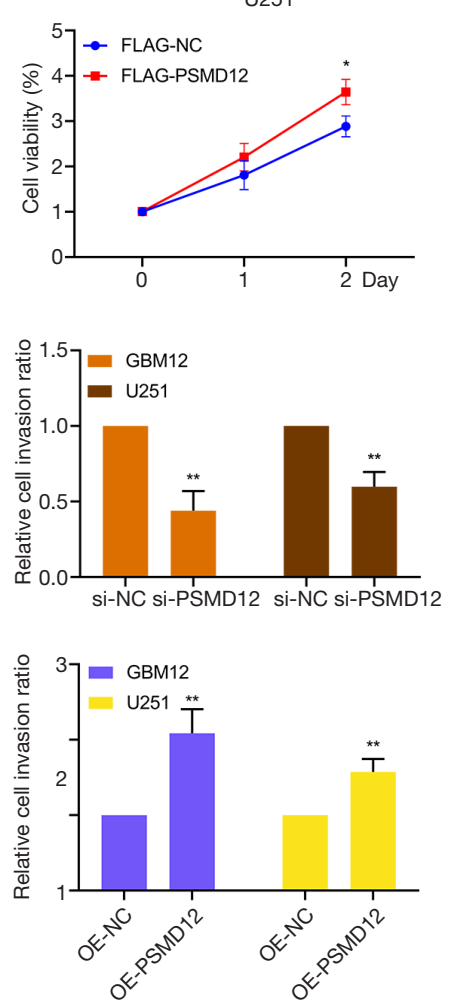

Figure 2 Proteasome 26S subunit, non-ATPase 12 (PSMD12) promotes the growth and invasion of glioma cells in vitro. (A) Knockdown efficiency of PSMD12 in glioblastoma 12 and U251 cells. (B) Transfection efficiency of PSMD12 in glioma cells. (C) Cell growth was detected by the Cell Counting Kit-8 (CCK-8) assay after PSMD12 knockdown in glioma lines. (D) Cell proliferation was measured by the CCK-8 assay after PSMD12 overexpression in glioma cell lines. (E) Invasion of glioma cells with PSMD12 knockdown was confirmed using the Transwell assay (magnification $\times 200$ ). Results from the quantitative analysis of the percentage of invading cells are shown. (F) Invasion of glioma cells with PSMD12 overexpression was determined using the Transwell assay (magnification $\times 200$ ). Results from the quantitative analysis of the percentage of invading cells are shown. Values are presented as the means \pm standard deviations from 3 independent experiments. *, $\mathrm{P}<0.05$; **, $\mathrm{P}<0.01$; and ${ }^{* * *}, \mathrm{P}<0.001$ compared with the control group.

\section{Knockdown of PSMD12 inhibits glioma tumor growth in vivo}

To further verify the antitumor effect of PSMD 12 knockdown on glioma in vivo, we generated GBM12 cells with stable PSMD12 knockdown and subcutaneously injected these cells into mice to construct a mouse xenograft model. The knockdown efficiency of lentivirus expressing sh-PSMD12 in GBM12 cells was measured by detecting the protein expression of PSMD12 by Western blotting. The results indicated that GBM12 cells stably knockdown PSMD12 were successfully established (Figure $3 A$ ). After we established the xenograft model by subcutaneous injection of the cells into mice, tumors developed over three weeks. 

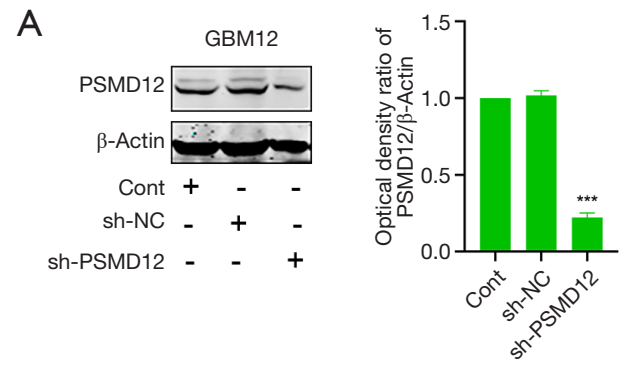

D

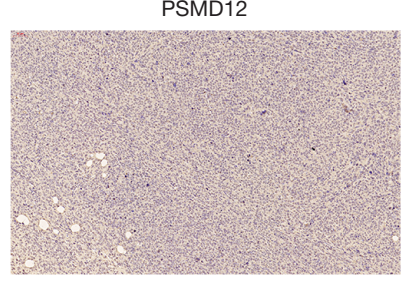

E

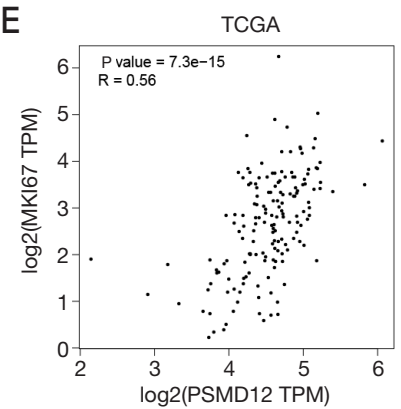

B

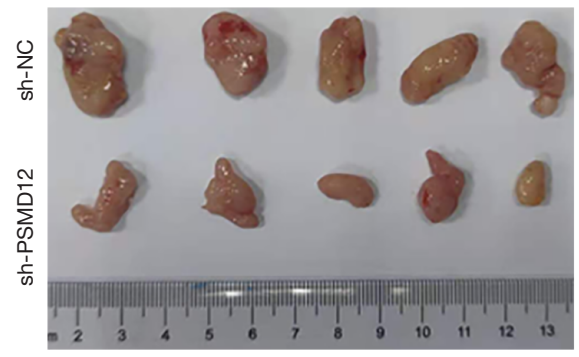

C

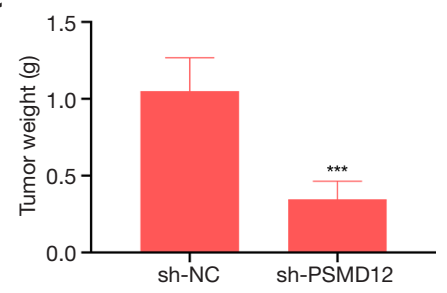

sh-NC Ki67
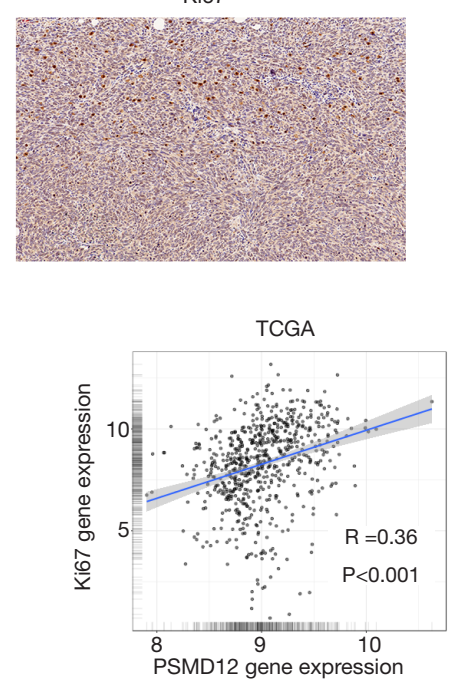

PSMD12

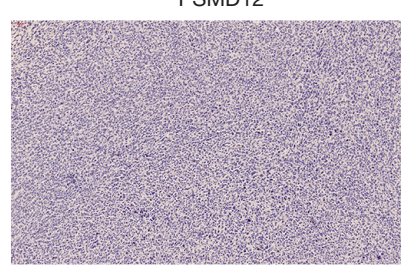

CGGA

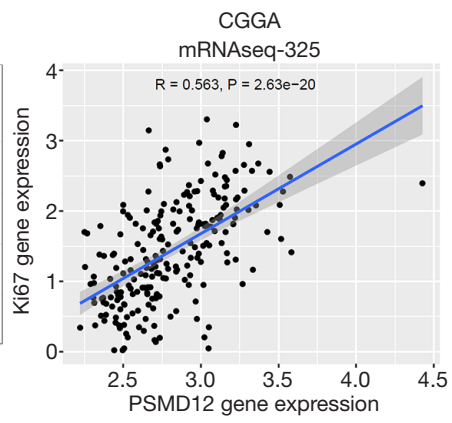

sh-PSMD12
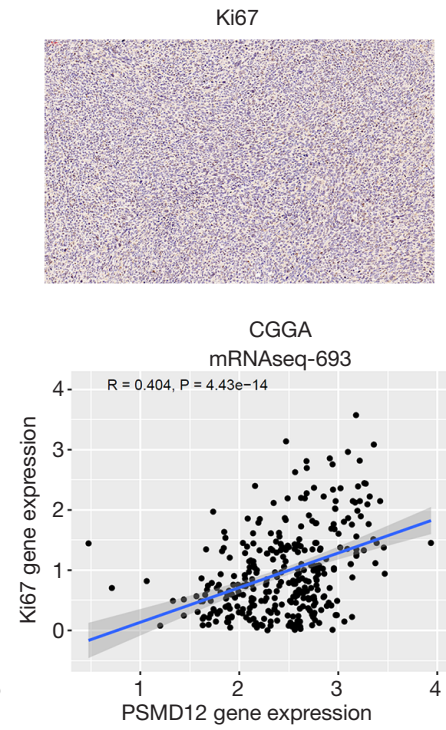

Figure 3 Knockdown of proteasome 26S subunit, non-ATPase 12 (PSMD12) inhibits glioma tumor growth in vivo. (A) Knockdown efficiency of PSMD12 was assessed by Western blotting of stable glioblastoma 12 (GBM12) cells. (B) Xenografts derived from shPSMD12 GBM12 cells (n=5). (C) Tumor weight of the sh-PSMD12 group was compared with that of the NC-PSMD12 group. (D) Immunohistochemistry was used to detect the expression of PSMD12 and Ki67 in xenograft tissue (magnification $\times 200$ ). (E) Analysis of the correlation of PSMD12 and Ki67 mRNA expression levels in the Gene Expression Profiling Interactive Analysis, GlioVis, and Chinese Glioma Genome Atlas databases. Values are presented as the means \pm standard deviations from 3 independent experiments. *** $\mathrm{P}<0.001$ compared with the control group.

We then removed the xenografts and found that the size and weight of tumor xenografts in the knockdown PSMD12 group were smaller than those in the control group, indicating that PSMD12 knockdown significantly inhibited xenograft growth (Figure 3B,C). Additionally, the IHC results showed that the Ki67 index of the xenografts in the sh-PSMD12 group was significantly lower than that of the xenografts in the sh-NC group (Figure 3D). These findings were further verified by the strong positive correlation between PSMD12 and Ki67 expression levels in TCGA and the CGGA databases (Figure 3E). Therefore, these data demonstrate that the knockdown of PSMD12 decreases the growth rate of glioma in vivo.

\section{PSMD12 regulation of Nrf2 expression and its positive correlation with Nrf2}

Our previous results indicated that PSMD12 can regulate the biological functions of glioma cells (Figures 2,3), but the specific molecular mechanism was still unknown. Accumulating evidence has revealed that $\mathrm{Nrf} 2$ can regulate the key signaling pathways of tumor cells and then participate in tumor progression by regulating the biological functions of tumor cells. Therefore, we investigated whether PSMD12 was involved in the regulation of $\mathrm{Nrf2}$. First, correlation analysis of PSMD12 and Nrf2 showed a significantly strong correlation at the mRNA level in TCGA 

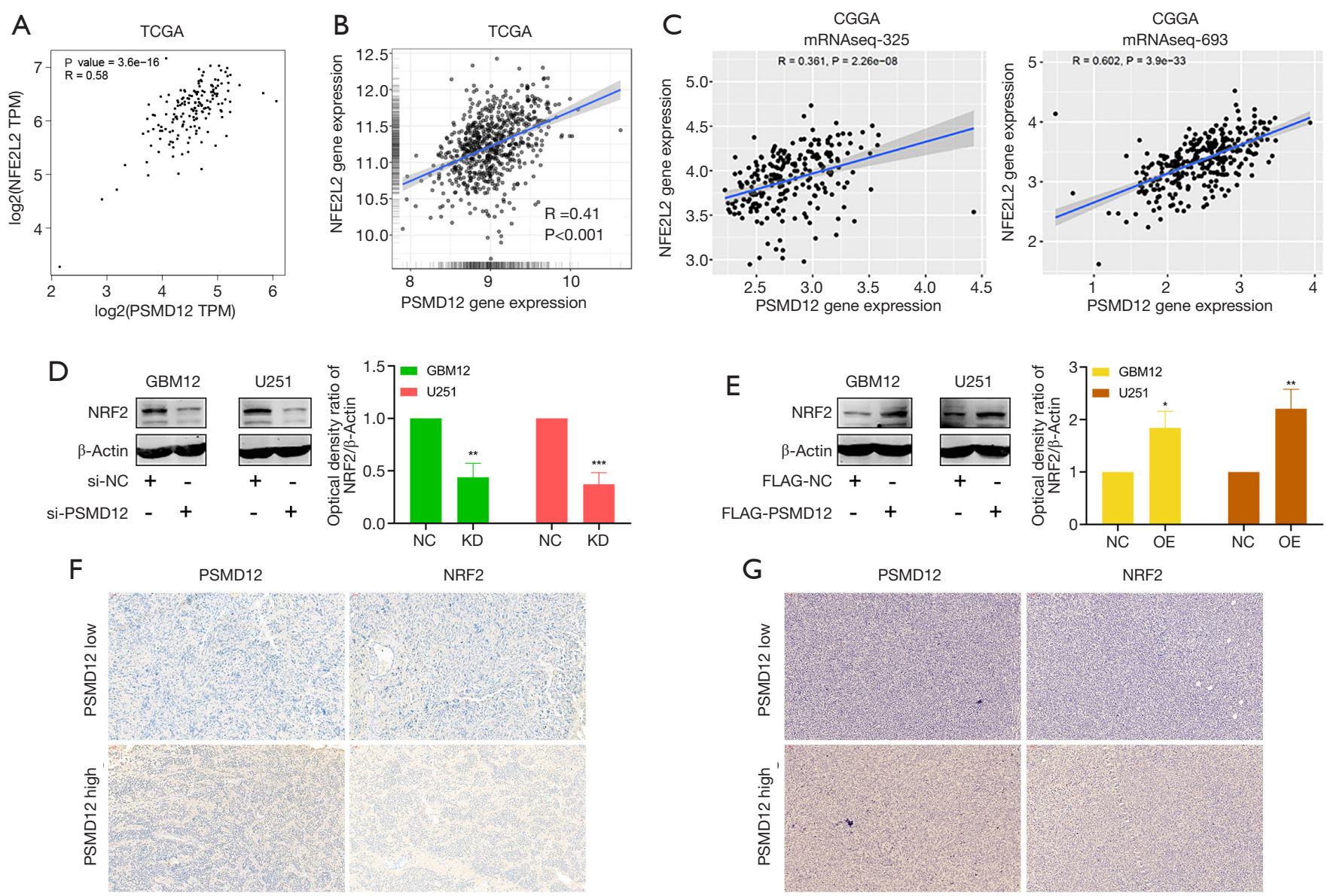

Figure 4 Proteasome 26S subunit, non-ATPase 12 (PSMD12) regulates the expression of nuclear factor E2-related factor 2 (Nrf2) and is positively correlated with Nrf2. (A,B,C) Analysis of the correlation between PSMD12 and Nrf2 mRNA expression levels from the Gene Expression Profiling Interactive Analysis, GlioVis, and Chinese Glioma Genome Atlas databases. (D,E) Western blotting was applied to detect the expression of Nrf2 after PSMD12 knockdown or PSMD12 overexpression in glioma cells. (F) PSMD12 and Nrf2 staining in glioma tissue by immunohistochemistry (IHC; magnification $\times 200$ ). (G) PSMD12 and Nrf2 staining in xenograft tissue by IHC (magnification $\times 200)$. Values are presented as the means \pm standard deviations from 3 independent experiments. ${ }^{*}, \mathrm{P}<0.05 ;{ }^{* *}, \mathrm{P}<0.01 ;$ and ${ }^{* * *}, \mathrm{P}<0.001$ compared with the control group.

and the CGGA databases (Figure 4A,B,C). Subsequently, we detected the protein expression level of Nrf2 in GBM12 and U251 cells after PSMD12 knockdown or overexpression. The Western blotting results showed that PSMD12 knockdown dramatically decreased the Nrf2 protein levels, while PSMD12 overexpression significantly increased the Nrf2 protein levels (Figure 4D,E). Alternatively, we used IHC to detect the expression of PSMD12 and Nrf2 in glioma tissues. Consistent with the results of TCGA and the CGGA databases, Nrf2 was also highly expressed in glioma tissues with a high expression of PSMD12, indicating that the expression of PSMD12 is positively correlated with that of Nrf2 (Figure 4F). The correlation of PSMD12 and Nrf2 was also verified by the IHC results from the xenograft model (Figure 4G). Collectively, these results indicate a strong positive correlation between PSMD12 expression and Nrf2 expression, and also indicate that PSMD12 may mediate glioma cell behavior by regulating $\mathrm{Nrf} 2$.

\section{Nrf2 plays an oncogenic role in glioma}

To explore the significance of Nrf2 in glioma, we first assessed the Nrf2 mRNA level in glioma versus normal tissues from TCGA and the CGGA databases. Consistent 


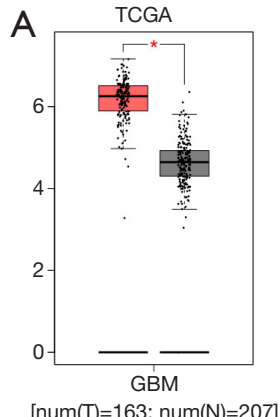

[num(T)=163; num $(N)=207$
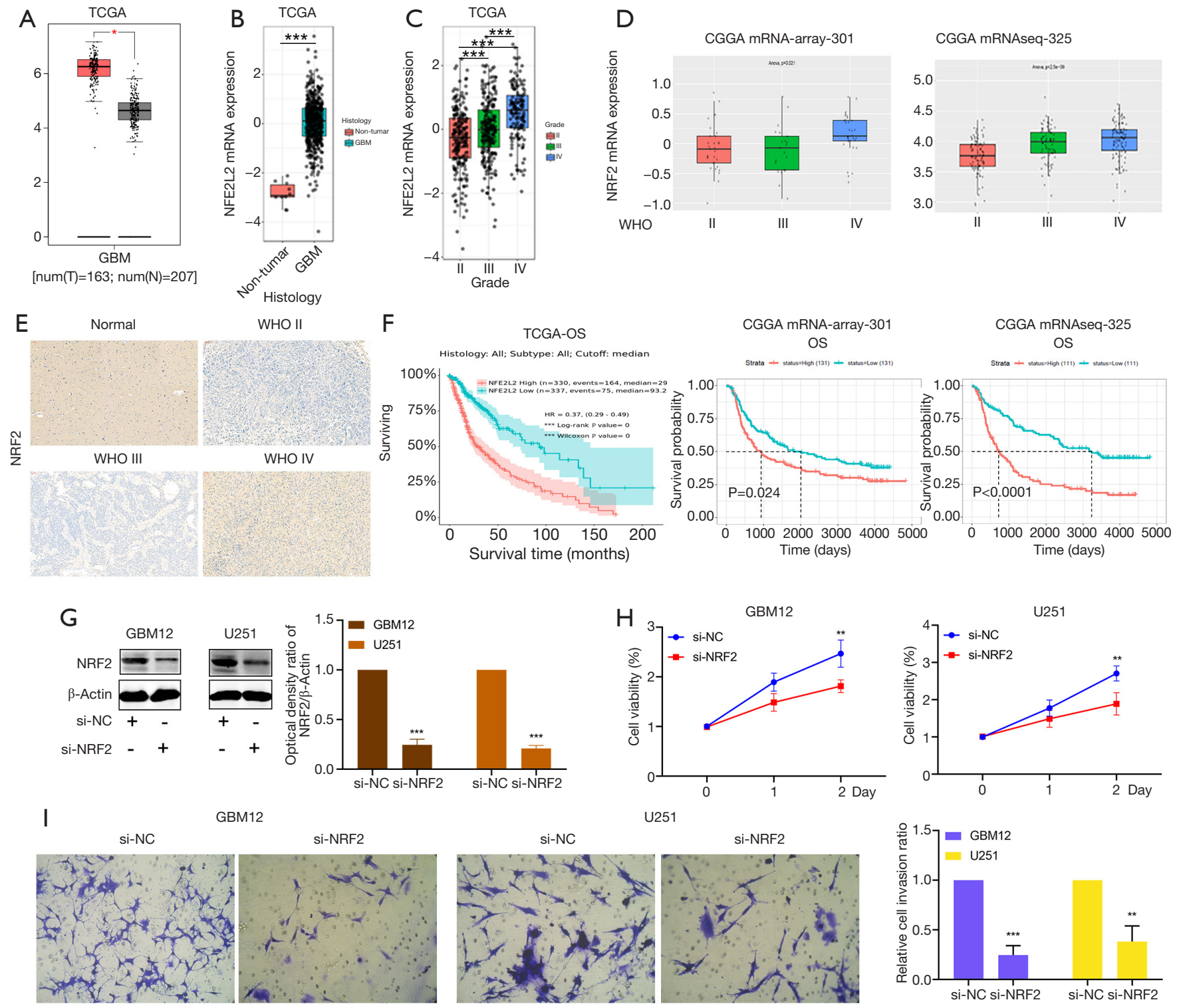

si-NRF2
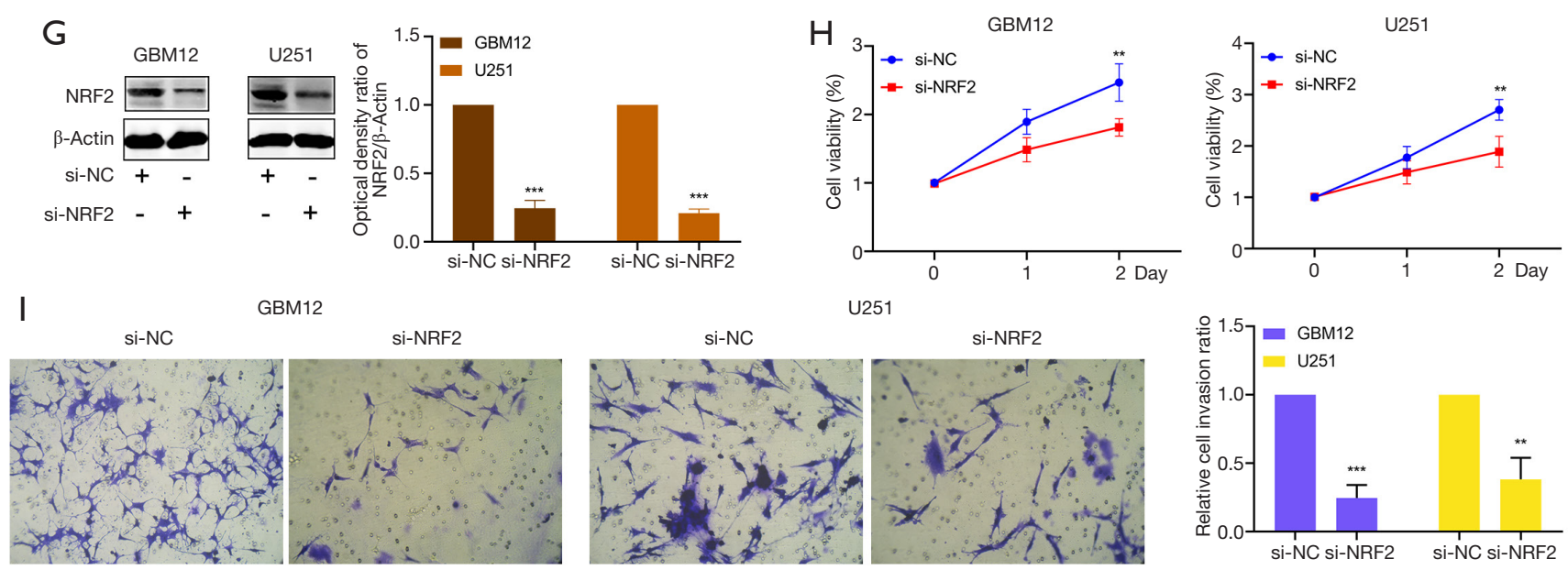

Figure 5 Nuclear factor E2-related factor 2 (Nrf2) plays an oncogenic role in glioma. (A,B,C,D) Relative levels of Nrf2 in glioma tissues and adjacent tissues from Gene Expression Profiling Interactive Analysis, GlioVis, and Chinese Glioma Genome Atlas (CGGA). (E) Expression of Nrf2 in glioma tissues and normal tissues by immunohistochemistry (magnification $\times 200$ ). (F) Kaplan-Meier analysis showed the overall survival of glioma patients with high/low Nrf2 expression from the GlioVis and CGGA websites. (G) Knockdown efficiency of Nrf2 in glioblastoma 12 and U251 cells. (H) Cell viability was determined by the Cell Counting Kit-8 assay after Nrf2 knockdown in glioma lines. (I) Invasion of glioma cells with Nrf2 knockdown was confirmed using the Transwell assay (magnification $\times 200$ ). Results from the quantitative analysis of the percentage of invading cells are shown. Values are presented as the means \pm standard deviations from 3 independent experiments. *, $\mathrm{P}<0.05 ;{ }^{* *}, \mathrm{P}<0.01$; and ${ }^{* * *}, \mathrm{P}<0.001$ compared with the control group.

with the expression pattern of PSMD12, the expression of Nrf2 was significantly higher in glioma tissues than in normal brain tissues and was also significantly higher in grade IV tumor tissues than in grade II and III tumor tissues (Figure $5 A, B, C, D$ ). These results were also verified by IHC analysis of samples from patients in our hospital (Figure 5E). Additionally, Kaplan-Meier analysis showed that glioma patients with high Nrf2 expression had a worse 

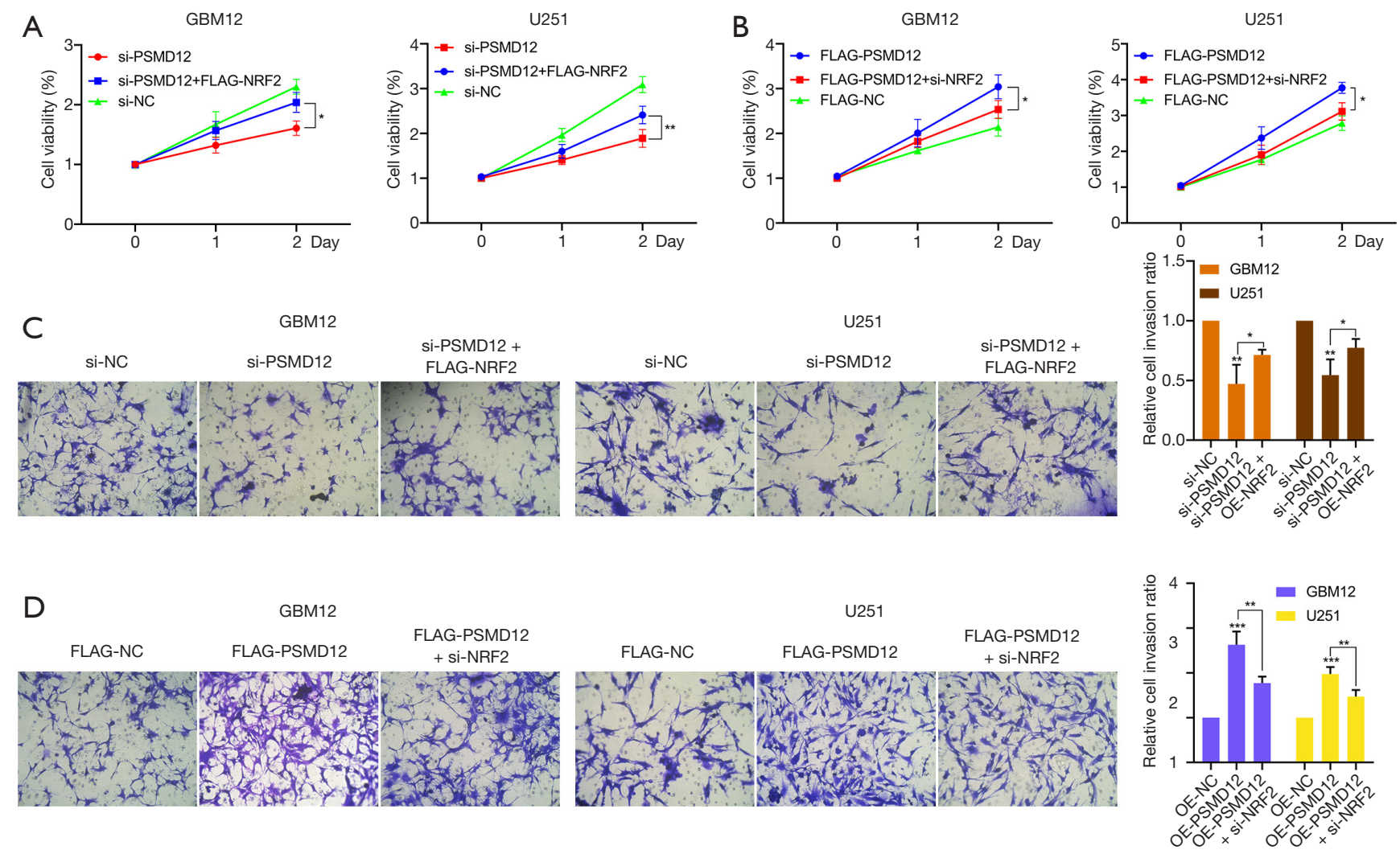

Figure 6 Proteasome 26S subunit, non-ATPase 12 (PSMD12) promotes aggressive behaviors in glioma cells by enhancing the expression of nuclear factor E2-related factor 2 (Nrf2). (A,B) Cell growth was assessed by the Cell Counting Kit-8 assay in PSMD12 knockdown or PSMD12-overexpressing cells with Nrf2 overexpression or Nrf2 knockdown. (C,D) Transwell assays were used to examine the invasion ability of glioma cells treated as described above (magnification $\times 200$ ). Results from the quantitative analysis of the percentage of invading cells are shown. Values are presented as the means \pm standard deviations from 3 independent experiments. *, $\mathrm{P}<0.05 ;{ }^{* *}, \mathrm{P}<0.01 ;$ and ${ }^{* * *}$, $\mathrm{P}<0.001$ compared with the control group.

prognosis than those with low Nrf2 expression in TCGA and the CGGA databases (Figure 5F). To further investigate the role of Nrf2 in glioma, we transfected glioma cells with Nrf2 siRNA to inhibit the protein expression of Nrf2. The Western blotting results showed that the Nrf2 knockdown efficiencies in GBM12 and U251 cells were $76 \%$ and $80 \%$, respectively (Figure 5G). The CCK-8 assay results showed that knockdown of $\mathrm{Nrf} 2$ markedly inhibited cell growth compared with that in the control group (Figure $5 H$ ). Furthermore, Transwell assays showed that the knockdown of Nrf2 significantly inhibited the invasion ability of the two glioma cell lines (Figure 5I). Therefore, these results revealed that the high expression of $\mathrm{Nrf} 2$ is associated with poor prognosis in glioma patients, and that Nrf2 knockdown and PSMD12 knockdown exhibit similar inhibitory effects on glioma.

\section{PSMD12 promotes aggressive behaviors in glioma cells by increasing the expression of $\mathrm{Nrf} 2$}

To investigate whether Nrf2 is involved in the PSMD12induced progression of glioma, we performed rescue experiments by overexpressing or inhibiting $\mathrm{Nrf} 2$ in PSMD12 knockdown or PSMD12-overexpressing cells, respectively. Compared with PSMD12 knockdown alone, PSMD12 knockdown combined with Nrf2 overexpression significantly promoted cell growth (Figure 6A). Similarly, the knockdown of Nrf2 in PSMD12-overexpressing cells significantly decreased the proliferation ability of glioma cells (Figure $6 B$ ). The cell invasion and cell proliferation results showed a similar trend (Figure 6C,D). These data indicate that PSMD12 may exhibit regulatory effects on glioma through the regulation of $\mathrm{Nrf} 2$. 


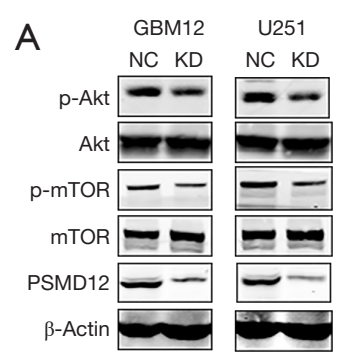

C

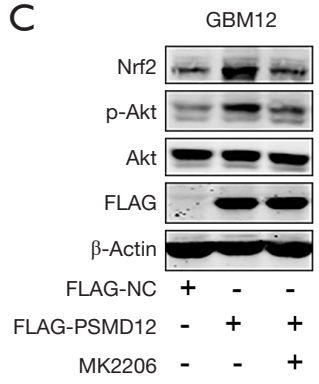

E

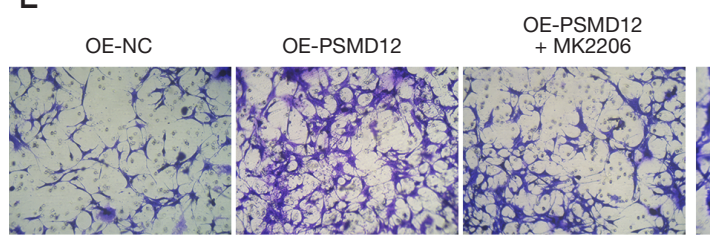

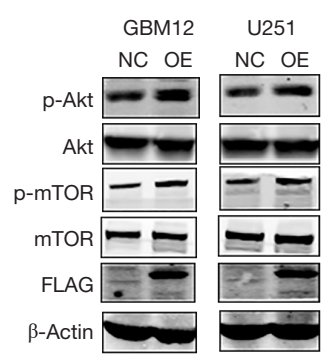

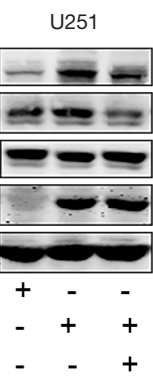

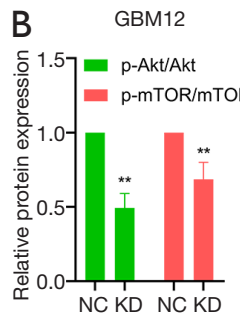

D
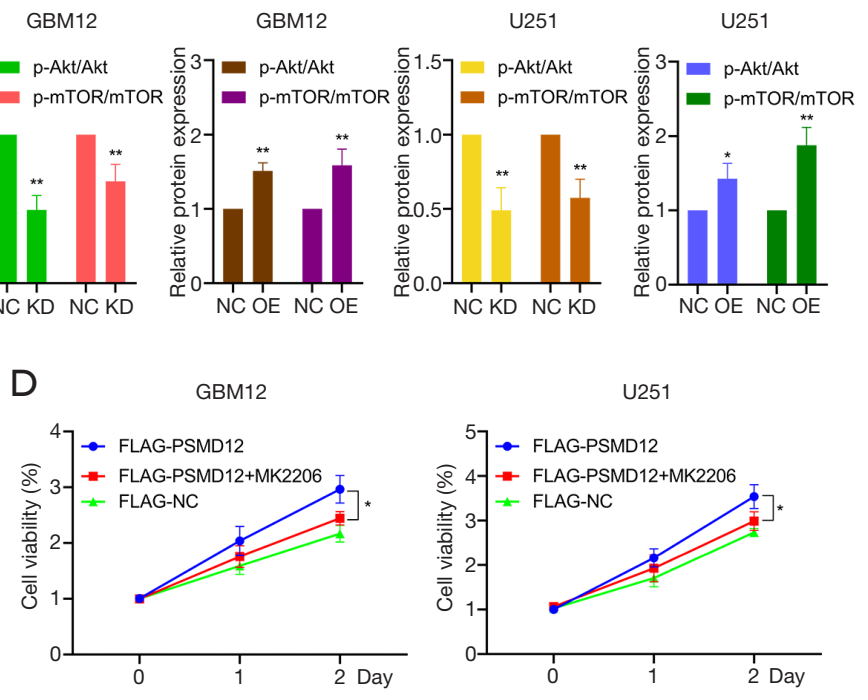

$\mathrm{U} 251$

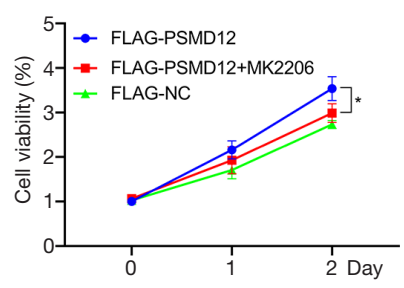

251
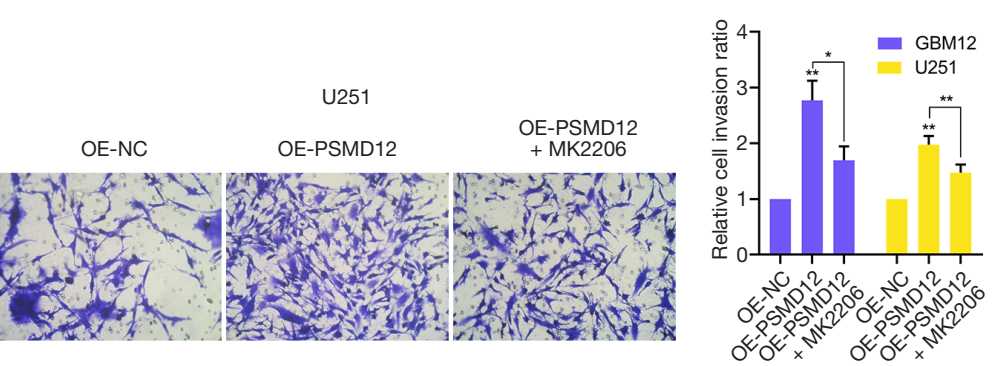

Figure 7 Akt pathway is required for the regulation of nuclear factor E2-related factor 2 (Nrf2) by proteasome 26S subunit, non-ATPase 12 (PSMD12) in glioma cells. (A) Levels of Akt/mTOR pathway proteins in PSMD12 knockdown and PSMD12-overexpressing cells by PSMD12 siRNA and overexpressed plasmid were detected using Western blotting. (B) Quantitative analysis of the band intensities of p-Akt, Akt, p-mTOR, and mTOR relative to that of -actin. (C) Levels Nrf2, p-Akt, and Akt proteins in PSMD12-overexpressing cells with or without MK2206 (2 $\mu \mathrm{M}, 18 \mathrm{~h}$ ) were detected using Western blotting. (D) Cell viability was assessed by the Cell Counting Kit-8 assay in glioma cells treated as described above. (E) Transwell assays were used to assess the invasion ability of glioma cells treated as described above (magnification $\times 200$ ). Results from the quantitative analysis of the percentage of invading cells are shown. Values are presented as the means \pm standard deviations from 3 independent experiments. *, $\mathrm{P}<0.05$; and ${ }^{* *}, \mathrm{P}<0.01$ compared with the control group.

\section{Akt pathway is required for the regulation of $\mathrm{Nrf2}$ by PSMD12 in glioma cells}

Akt signaling can participate in tumor cell proliferation, apoptosis, and chemotherapy resistance by regulating the expression of Nrf2 (15-17). Furthermore, Akt signaling plays a vital role in the occurrence and development of various tumors $(18,19)$. Therefore, we speculate that PSMD12 regulates the expression of Nrf2 through the Akt signaling pathway. To verify this, we first assessed the activation of the Akt pathway in glioma cell lines with knockdown and overexpression of PSMD12. The results showed that the levels of p-Akt and p-mTOR (mammalian target of rapamycin) in GBM12 and U251 cells were significantly increased in the PSMD12 knockdown group compared with the control group (Figure $7 A, B$ ). In contrast, PSMD12 overexpression markedly increased the levels of p-Akt and p-mTOR (Figure 7A,B). To assess whether Akt signaling plays an important role in the regulation of $\mathrm{Nrf} 2$ by PSMD12, Western blotting, CCK-8, and Transwell assays were performed with PSMD12-overexpressing cells treated with MK2206, a specific Akt pathway inhibitor. The Western blotting results showed that MK2206 treatment significantly reversed the upregulation of $\mathrm{Nrf2}$ protein levels in PSMD12-overexpressing cells (Figure 7C). Similarly, MK2206 treatment significantly reduced the pro- 
proliferation and pro-invasion effects induced by PSMD12 overexpression in glioma cells (Figure $7 D, E$ ).

\section{Discussion}

Glioma is the most common and most fatal malignant tumor among adult central nervous system tumors (20). Although the prognosis of glioma patients can be improved by various clinical treatments, such as surgery, radiotherapy, and chemotherapy, its recurrence and mortality rates are still high due to its high aggressiveness and high proliferation rate (21). Therefore, the identification of novel therapeutic targets has become the focus of glioma research. Previous studies have demonstrated that PSMD12 plays an oncogenic role in prostate cancer and breast cancer, but its role in glioma and its underlying molecular mechanisms remain unclear. In our study, the elevated expression of PSMD12 was observed in glioma tissues compared with normal tissues, especially in high-grade glioma tissue, and high PSMD12 expression was found to be associated with poor prognosis. In addition, PSMD12 knockdown significantly inhibited cell proliferation and invasion in vitro and glioma xenograft growth in vivo. The overexpression of PSMD12 exhibited the opposite effects. Furthermore, the expression of PSMD12 was positively correlated with the expression of $\mathrm{Nrf2}$, and $\mathrm{Nrf} 2$ overexpression reversed the inhibition of cell growth and invasion induced by PSMD12 knockdown. Finally, the upregulation of PSMD12 in glioma cells promoted the expression of the Nrf2 protein by activating Akt signaling.

The UPS is a very large and extremely complex system in the cell, and involves ubiquitin, Ub-activating enzymes, Ub-conjugating enzymes, Ub-protein ligases, and the $26 \mathrm{~S}$ proteasome complex. It plays a vital role in protein degradation, protein conversion, and ubiquitinationmediated signal transduction $(22,23)$. Ubiquitination is a post-translational epigenetic modification that exerts its degradation function through the following three key enzymes: activating enzymes, ligases, and conjugating enzymes (22). Previous studies have shown that the UPS plays a vital role in biological processes, including apoptosis, cell proliferation, signal transduction, and DNA damage, by controlling the expression of key proteins in the cell cycle, tumorigenesis, and development $(24,25)$. This is similar to the function of PSMD12 in cells $(5,6)$. PSMD12, a non-ATPase subunit of the $19 \mathrm{~S}$ regulator of the $26 \mathrm{~S}$ proteasome complex (the core component of the UPS), plays an important biological role in neurodevelopment and neurogenesis $(4,22)$. In recent years, PSMD12 has been increasingly studied in the field of cancer; for example, PSMD12 can promote the progression of breast cancer by inhibiting the expression of proapoptotic genes, such as thioredoxin (Trx)-interacting protein (TXNIP), growth arrest and DNA damage inducible protein 45 alpha (GADD45A) and growth arrest and DNA damage inducible protein 45 beta (GADD45B) (8). Our study showed that PSMD12 knockdown markedly suppressed glioma cell growth and invasion in vitro and reduced the tumorigenicity of glioma cells in vivo. We first reported that PSMD12 is upregulated in glioma tissues compared with normal brain tissues, and is positively correlated with glioma grade (Figure 1). More importantly, glioma patients with low PSMD12 expression had better OS than patients with high PSMD12 expression, which is similar to the results for PSMD12 in breast cancer (8). These results suggest that PSMD12 functions as an oncogene in tumor development, but further studies are needed to confirm its role.

Nrf2 is not only an important transcription factor for a variety of anti-inflammatory enzymes and antioxidant factors but is also one of the transcription factors involved in tumor cell survival and tumor progression (26). An increasing number of studies have shown that the expression of Nrf2 is significantly increased in a variety of solid tumors, such as breast cancer (27), stomach cancer (28), head and neck cancer (29), and ovarian cancer (30), and that the protein is transferred into the nucleus to induce the transcriptional activation of tumor-protective genes to protect cells from apoptosis under oxidative stress, thereby promoting tumor progression (31). In addition, Nrf2 and p62 can also interact with each other through other signaling pathways to promote cell proliferation and chemotherapy resistance and other biological behaviors. For example, the phosphorylation of p62 promotes the expression and nuclear translocation of $\mathrm{Nrf} 2$, and the nuclear entry of $\mathrm{Nrf} 2$ in turn increases the transcription and translation of $\mathrm{p} 62$, which forms a positive feedback loop preventing apoptosis (32). Furthermore, esophageal cancer cells with a high expression of Nrf2 can effectively promote epithelial-mesenchymal transition (EMT)-driven cell metastasis (33). Therefore, the abnormal activation of $\mathrm{Nrf} 2$ in tumors leads to poor prognosis in patients $(34,35)$. In our study, we found that Nrf2 was markedly upregulated in glioma samples, and that glioma patients with high PSMD12 expression had worse OS than those with low PSMD12 expression. Moreover, Nrf2 knockdown significantly suppressed the proliferation and invasion of 
glioma cells, which was consistent with the role of $\mathrm{Nrf} 2$ in other solid tumors. Under normal physiological conditions, Nrf2 and Keap1 can be targeted for ubiquitination by E3 ligases and can be degraded through the $26 \mathrm{~S}$ proteasome pathway (36); PSMD12 is an important regulatory subunit of the $26 \mathrm{~S}$ proteasome (22). Therefore, we speculated that there is a correlation between PSMD12 and Nrf2. To verify this, we analyzed bioinformatics data, patient samples, and xenograft tissues and found that the expression of PSMD12 in glioma has a strong positive correlation with that of $\mathrm{Nrf} 2$. More importantly, a rescue experiment revealed that $\mathrm{Nrf} 2$ overexpression could alleviate or even reverse the inhibitory effect of PSMD12 knockdown on the biological behavior of glioma, which indicates that Nrf2 functions as a potential downstream target of PSMD12. Studies have reported that the Akt/mTOR pathway is abnormally activated in a variety of tumor cells and is involved in regulating tumor cell proliferation, apoptosis, and $\operatorname{EMT}(17,18)$. Interestingly, Akt signaling can participate in tumor cell proliferation, apoptosis, and chemotherapy resistance by regulating the expression of Nrf2 (15-17). Consistent with these results, our study showed that PSMD12 promotes the activation of the Akt/mTOR pathway and subsequently enhances Nrf2 expression. The inhibition of Akt phosphorylation had an inhibitory effect on cell growth and invasion following PSMD12 overexpression, which implies that the Akt pathway might be an essential signaling pathway by which PSMD12 functions in glioma.

In summary, we found that PSMD12 and Nrf2 function as tumor promoters in glioma and that both could accelerate the development and progression of glioma by regulating cell invasion and proliferation. Moreover, Nrf2 expression, regulated by the Akt pathway, contributes to PSMD12induced glioma progression. Overall, the regulation of glioma by PSMD12 through Nrf2 may reveal new and precise targets for the treatment of glioma.

However, more studies are still needed to identify the oncogenic role of PSMD12 in glioma, such as the specific mechanism by which PSMD12 regulates the Akt pathway, the potential mechanism of PSMD12 regulating Nrf2 and mediating Nrf2 entry into the nucleus, and the cell specificity of these effects etc. Only under these circumstances can we better understand the tumourpromoting effect of PSMD12.

\section{Acknowledgments}

Funding: This work was supported by the Project of Suzhou
Health Talents, China (No. 2020090) and the Youth Medical Doctors Project of Jiangsu Province, China (No. QNRC2016870).

\section{Footnote}

Reporting Checklist: The authors have completed the ARRIVE reporting checklist. Available at http://dx.doi. org/10.21037/atm-21-1481

Data Sharing Statement: Available at http://dx.doi. org/10.21037/atm-21-1481

Conflicts of Interest: All authors have completed the ICMJE uniform disclosure form (available at http://dx.doi. org/10.21037/atm-21-1481). The authors have no conflicts of interest to declare.

Ethical Statement: The authors are accountable for all aspects of the work in ensuring that questions related to the accuracy or integrity of any part of the work are appropriately investigated and resolved. All procedures performed in this study involving human participants were in accordance with the Declaration of Helsinki (as revised in 2013). All human participants involved in this study provided informed consent. The animal experiment complied with the principles of the Animal Centre of the Second Affiliated Hospital of Soochow University.

Open Access Statement: This is an Open Access article distributed in accordance with the Creative Commons Attribution-NonCommercial-NoDerivs 4.0 International License (CC BY-NC-ND 4.0), which permits the noncommercial replication and distribution of the article with the strict proviso that no changes or edits are made and the original work is properly cited (including links to both the formal publication through the relevant DOI and the license). See: https://creativecommons.org/licenses/by-nc-nd/4.0/.

\section{References}

1. Ostrom QT, Bauchet L, Davis FG, et al. The epidemiology of glioma in adults: a "state of the science" review. Neuro Oncol 2014;16:896-913.

2. Ohgaki H, Kleihues P. Epidemiology and etiology of gliomas. Acta Neuropathol 2005;109:93-108.

3. Bush NA, Chang SM, Berger MS. Current and future strategies for treatment of glioma. Neurosurg Rev 
2017;40:1-14.

4. Zhang N, Osborn M, Gitsham P, et al. Using yeast to place human genes in functional categories. Gene 2003;303:121-9.

5. Küry S, Besnard T, Ebstein F, et al. De Novo Disruption of the Proteasome Regulatory Subunit PSMD12 Causes a Syndromic Neurodevelopmental Disorder. Am J Hum Genet 2017;100:689.

6. Wang T, Jiang X, Chen G, et al. Interaction of amyotrophic lateral sclerosis/frontotemporal lobar degeneration-associated fused-in-sarcoma with proteins involved in metabolic and protein degradation pathways. Neurobiol Aging 2015;36:527-35.

7. Kohrt SE, Awadallah WN, Phillips RA 3rd, et al. Identification of Genes Required for Enzalutamide Resistance in Castration-Resistant Prostate Cancer Cells In Vitro. Mol Cancer Ther 2021;20:398-409.

8. Du X, Shen X, Dai L, et al. PSMD12 promotes breast cancer growth via inhibiting the expression of proapoptotic genes. Biochem Biophys Res Commun 2020;526:368-74.

9. Tonelli C, Chio IIC, Tuveson DA. Transcriptional Regulation by Nrf2. Antioxid Redox Signal 2018;29:1727-45.

10. Taguchi K, Yamamoto M. The KEAP1-NRF2 System in Cancer. Front Oncol 2017;7:85.

11. Pölönen P, Jawahar Deen A, Leinonen HM, et al. Nrf2 and SQSTM1/p62 jointly contribute to mesenchymal transition and invasion in glioblastoma. Oncogene 2019;38:7473-90.

12. Kanamori M, Higa T, Sonoda Y, et al. Activation of the NRF2 pathway and its impact on the prognosis of anaplastic glioma patients. Neuro Oncol 2015;17:555-65.

13. Fan Z, Wirth AK, Chen D, et al. Nrf2-Keap1 pathway promotes cell proliferation and diminishes ferroptosis. Oncogenesis 2017;6:e371.

14. Wang Z, Li Z, Wu Q, et al. DNER promotes epithelialmesenchymal transition and prevents chemosensitivity through the $\mathrm{Wnt} /$ beta-catenin pathway in breast cancer. Cell Death Dis 2020;11:642.

15. Chen X, Wu Q, Chen Y, et al. Diosmetin induces apoptosis and enhances the chemotherapeutic efficacy of paclitaxel in non-small cell lung cancer cells via Nrf2 inhibition. Br J Pharmacol 2019;176:2079-94.

16. Su J, Zhang F, Li X, et al. Osthole promotes the suppressive effects of cisplatin on NRF2 expression to prevent drug-resistant cervical cancer progression. Biochem Biophys Res Commun 2019;514:510-7.
17. Zhang XQ, Yao C, Bian WH, et al. Effects of Astragaloside IV on treatment of breast cancer cells execute possibly through regulation of $\mathrm{Nrf} 2$ via $\mathrm{PI} 3 \mathrm{~K} / \mathrm{AKT} / \mathrm{mTOR}$ signaling pathway. Food Sci Nutr 2019;7:3403-13.

18. Li Z, Li C, Wu Q, et al. MEDAG enhances breast cancer progression and reduces epirubicin sensitivity through the AKT/AMPK/mTOR pathway. Cell Death Dis 2021;12:97.

19. Wang Z, Zhu Z, Li C, et al. NMIIA promotes tumorigenesis and prevents chemosensitivity in colorectal cancer by activating AMPK/mTOR pathway. Exp Cell Res 2021;398:112387.

20. Molinaro AM, Taylor JW, Wiencke JK, et al. Genetic and molecular epidemiology of adult diffuse glioma. Nat Rev Neurol 2019;15:405-17.

21. Ma Q, Long W, Xing C, et al. Cancer Stem Cells and Immunosuppressive Microenvironment in Glioma. Front Immunol 2018;9:2924.

22. Khalil R, Kenny C, Hill RS, et al. PSMD12 haploinsufficiency in a neurodevelopmental disorder with autistic features. Am J Med Genet B Neuropsychiatr Genet 2018;177:736-45.

23. Stewart MD, Ritterhoff T, Klevit RE, et al. E2 enzymes: more than just middle men. Cell Res 2016;26:423-40.

24. Scholz N, Kurian KM, Siebzehnrubl FA, et al. Targeting the Ubiquitin System in Glioblastoma. Front Oncol 2020;10:574011.

25. Yang H, Chen X, Li K, et al. Repurposing old drugs as new inhibitors of the ubiquitin-proteasome pathway for cancer treatment. Semin Cancer Biol 2021;68:105-22.

26. Panieri E, Buha A, Telkoparan-Akillilar P, et al. Potential Applications of NRF2 Modulators in Cancer Therapy. Antioxidants (Basel) 2020;9:193.

27. Almeida M, Soares M, Ramalhinho AC, et al. The prognostic value of NRF2 in breast cancer patients: a systematic review with meta-analysis. Breast Cancer Res Treat 2020;179:523-32.

28. Jeddi F, Soozangar N, Sadeghi MR, et al. Nrf2 overexpression is associated with $\mathrm{P}$-glycoprotein upregulation in gastric cancer. Biomed Pharmacother 2018;97:286-92.

29. Roh JL, Jang H, Kim EH, et al. Targeting of the Glutathione, Thioredoxin, and Nrf2 Antioxidant Systems in Head and Neck Cancer. Antioxid Redox Signal 2017;27:106-14.

30. Bao L, Wu J, Dodson M, et al. ABCF2, an Nrf2 target gene, contributes to cisplatin resistance in ovarian cancer cells. Mol Carcinog 2017;56:1543-53.

31. Wang XJ, Hayes JD, Wolf CR. Generation of a stable 
antioxidant response element-driven reporter gene cell line and its use to show redox-dependent activation of nrf2 by cancer chemotherapeutic agents. Cancer Res 2006;66:10983-94.

32. Sengupta A, Mukherjee S, Ghosh S, et al. Partial impairment of late-stage autophagic flux in murine splenocytes leads to sqstm1/p62 mediated nrf2-keap1 antioxidant pathway activation and induced proteasomemediated degradation in malaria. Microb Pathog 2020;147:104289.

33. Shen H, Yang Y, Xia S, et al. Blockage of Nrf2 suppresses the migration and invasion of esophageal squamous cell carcinoma cells in hypoxic microenvironment. Dis

Cite this article as: Wang Z, Li Z, Xu H, Liao Y, Sun C, Chen Y, Sheng M, Lan Q, Wang Z. PSMD12 promotes glioma progression by upregulating the expression of Nrf2. Ann Transl Med 2021;9(8):700. doi: 10.21037/atm-21-1481
Esophagus 2014;27:685-92.

34. Moon EJ, Giaccia A. Dual roles of NRF2 in tumor prevention and progression: possible implications in cancer treatment. Free Radic Biol Med 2015;79:292-9.

35. Hamada S, Taguchi K, Masamune A, et al. Nrf2 promotes mutant K-ras/p53-driven pancreatic carcinogenesis. Carcinogenesis 2017;38:661-70.

36. Itoh K, Ye P, Matsumiya T, et al. Emerging functional cross-talk between the Keap1-Nrf2 system and mitochondria. J Clin Biochem Nutr 2015;56:91-7.

(English Language Editor: R. Scott) 